\title{
A NOVEL MICRO-SCALE RECOMBINING TECHNIQUE USING LATERAL JOINING FOR A LARGE-AREA MOLDING WITH SMALL FEATURES
}

\author{
Chih-Wei Wu, Long-Sun Huang, Jer-Haur Chang*, Sen-Yeu Yang*, Chih-Kung Lee \\ Institute of Applied Mechanics, National Taiwan University \\ * Department of Mechanical Engineering, National Taiwan University \\ No. 1, Sec. 4, Roosevelt Rd., Taipei 106, Taiwan R.O.C. \\ Phone: 886-2-33665653, Fax: 886-2-33665639, E-mail: $\underline{\text { shuang@mems.iam.ntu.edu.tw }}$
}

\begin{abstract}
ABSTRATE
A novel micro-scale recombining technique using a lateral joining of silicon crystal planes is first presented. The new concept of the technique is realized by fabricating a large silicon molding plate beyond a wafer size with small features. The replication of the joint plate using the hot embossing technqiue has been characterized in surface profiles, pictures, and optical performance. Both experiment and simulation results reached good agreement in diffractive optical intensity. Furthermore, the scalability of the technique was extensively demonstrated with three silicon wafers. As a result, the novel technique provides a potentially low-cost approach to fill the technology gap between the conventional precision machining and photolithography-based micromachining for a beyond-wafer-size silicon plate with small features.
\end{abstract}

\section{INTRODUCTION}

The commercialization of microsystem technology requires low-cost, high-volume production microfabrication methods. Use of polymer or replcated plastic microfabrication is becoming attractive for low-cost alternatives in increasingly growing applications of disposable, biocompatible devices and high-performacne micro optical elements [1-3]. Among production and low-cost methods of replication is a hot embossing process that may transfer delicate microstrutures onto a plastic film. Since the market size is still far small compared to the electronic semicoductor industry, most microstructure-based devices commercially manufactured in a size of 4- or 6-inch wafers are sufficient to meet the demand. Less interest drives the MEMS technique into a larger wafer size up to 8-inch or even 12-inch. A new demand of a large molding plate with microstructures is present for backlight module applications in liquid crystal display. The low-cost solution has not been available yet.

In current microfabrication, the means that employs the photolithographic method for micro scale features is limited in its wafer size. Nevertheless, the conventional machining techniques capable of manufacturing beyond-100 $\mu \mathrm{m}$ structures on a beyond-wafer-size plate pose severe challenges in fabricating micro features.

The recombining technique is a choice that uses a continuous stamping hot-embossed approach like a stepper to replicate patterns onto a very large substrate. Meanwhile, the pattern-transferred substrate is required to lie on a costly, precisely controlled moving stage. However, it is normally found with reqular equipment that the problem of undesired, irregular patterns arises in misalignment between stamping steps.

The paper presents a new approach that is intended to expand the technology limitation for a large beyond-a-wafer-size plate with micro-scale features. A novel micro-scale recombining technique using a lateral joining of anisotropically bulk-micromachined crystal planes is first presented. The new concept of the technique is to fabricate a large plate beyond-a-wafer-size with small features for molding, as schematically shown in Fig. 1.

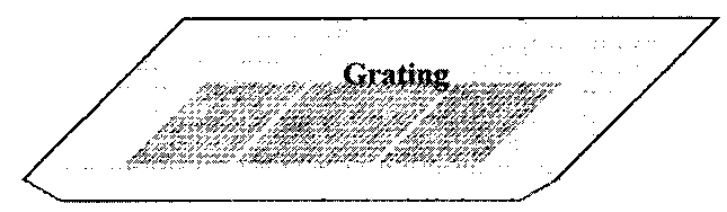

Fig. 1 A schematic of joined silicon wafers

\section{FABRICATION}

Anisotropic bulk-micromachining and a lateral joining of Si bulk-micromachined crystal planes are key to realize the concept. Fig. 2 shows the fabrication process of the joining wafers. The fabrication begins with two double-side polished silicon wafers deposited with $1000 \AA$ silicon dioxide and $1500 \AA$ silicon nitride. The films are used as an anisotropic masking layer, and reactive ion etching (RIE) is then followed to selectively etch away the masking layer. After the anisotropic bulk-micromachining etches through the entire wafers, the slanted crystal planes of two wafers are made ready. The microstructures of grating is then manufactured upon the processed square wafers. The reactive ion etching is used to form micro grating structures on a processed wafer.

It is noted that the photoresist spin coating over surfaces encountered difficulty of uniformity at the 
perimeter of the processed edge-sharp wafer. Serious cares of the delicate process need to be taken.

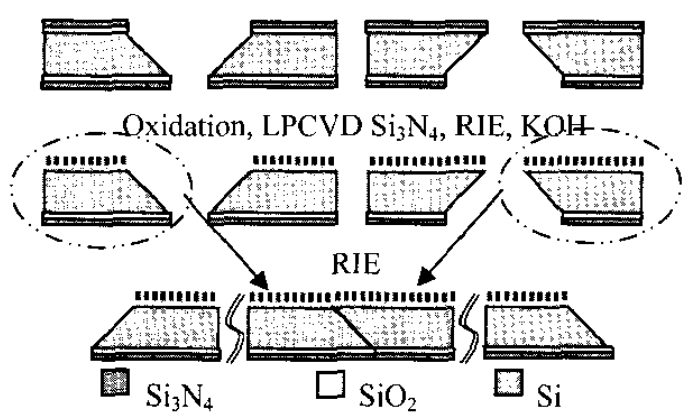

Fig. 2 Fabrication process

\section{JOINING TECHNIQUE}

The critical aspect of the lateral wafer joining technique is to maintain two wafers with same height, minimal tilting, and seamless connection. To keep two joined wafers on the same plane, a flat surface of a polished $\mathrm{Si}$ wafer is used as a reference plane. As shown in Fig. 3 (a) and (b) of the top and cross-sectional views, two laterally joined wafers are clamped with the flat reference surfaces around the perimeter. External clips are applied to bring intimate contact between the joined wafers and reference plates. With the clamping, the joined wafers may keep its height and tilting as the same plane as possible.. In addition, as shown in Fig. 3 (a), external lateral force is exerted at one side of the wafer to minimize the interface gap for seamless joining. Once the joint wafers are tightened, epoxy resin is then used to physically bond the wafers together. As a result of the bonding, all the subsequent handling and hot embossing processes are then relaxed to maintain their tilting, same plan, and seamless joining.

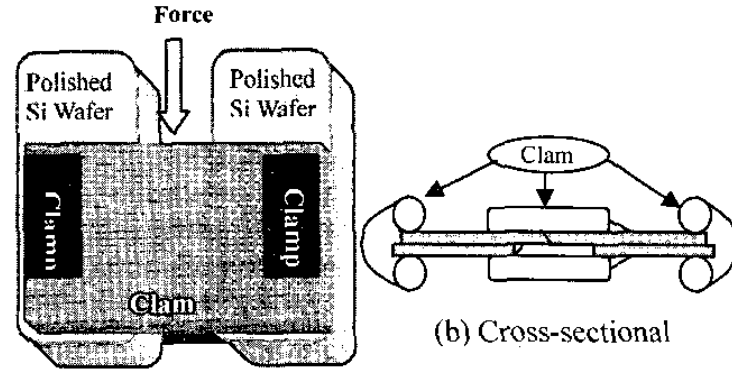

(a) Top

Fig. 3 Clamping of two joined wafers at their perimeters with steel clips

\section{MICRO HOT EMBOSSING REPLICATING TECHNOLOGY}

The hot embossing process is a production, low-cost method for the replication of precision micro-structures. Replication by hot embossing involves pressing the two joined wafers against a plastic sheet at a temperature above the softening point of the plastic substrate. Polyvinylchloride(PVC) film is often used for most replication work since it has lower $\mathrm{Tg}$ whose glass transition temperature is $87^{\circ} \mathrm{C}$. Its is also suitable for micro optic elements. The experiment was carried out at embossed temperature $90^{\circ} \mathrm{C}$ and pressure $30 \mathrm{kgf} / \mathrm{cm}^{2}$.

Although a silicon wafer itself is brittle, the mold that successfully utilized silicon material [1] in a hot embossing technique was reported. The material is most suitable for molded optic elements or regular microstructures. As depicted in Fig. 4, a typical hot embossing molding consists of heating and cooling systems, a pressing stamper, and a soft pad that is often required to cushion pressurized samples against pressing heating plate. The procedure to achieve a hot embossing process starts with a sample-suctioned holding. A pre-embossing contact is applied, followed by a programmed heating and thus increasing pressure. Once patterns are replicated, relaxation and de-molding are then to complete a hot embossing procedure.

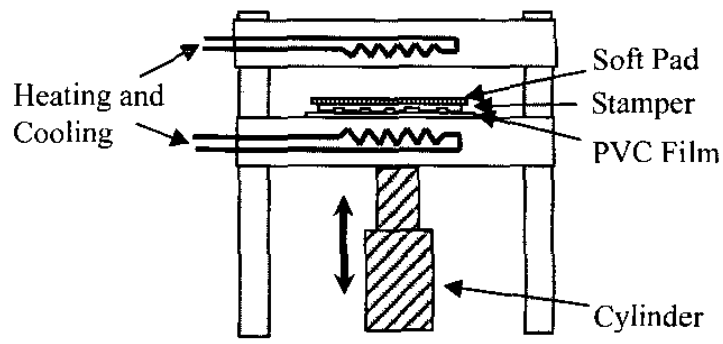

Fig. 4 Hot embossing process

\section{DISCUSSION}

The joint $\mathrm{Si}$ plate was characterized to investigate its feasibility. The measurement of the orginal master and its replciated structures was carried out at the interface of their surface profiles, pictures, and its associated optical performance.

As for the crevice at the interface, the external force was exerted in one side of the outside wafer to minimize the connected gap as shown in Fig. 5. Fig. 5 also indicates the surface profile of two joined wafers which achieves virtually same height of 
sub-micron discrimination of $6989 \AA$. As the exerted force was applied toward inside of one wafer, the gap can be reduced to achieve around 5 6 $\mu \mathrm{m}$. The approach achieves virtually seamless joining while traditional precision machining techniques hardly achieve such a result in an order of several microns.

With such significant results, the approach has ensured the conceptual feasbility of the lateral joining using anisotropically bulk-micromachined crystal planes of silicon wafers.

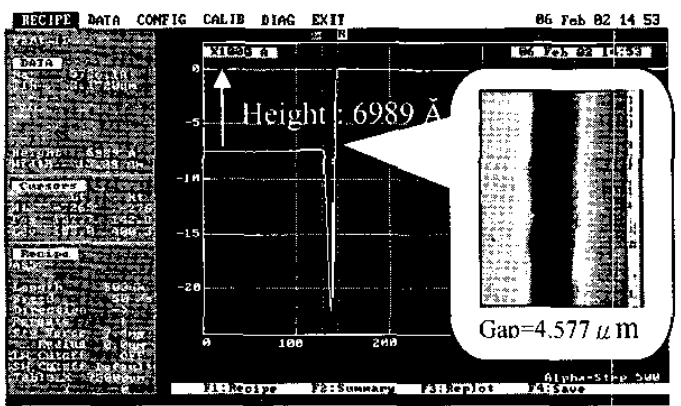

Fig. 5 Measured height at the interface of two joined wafers and the SEM picture of the crevice

Fig. 6 shows that the micro grating structures in a pitch of $4 \mu \mathrm{m}$ have been successfully manufactured at the sharp edge of the wafer. Serious care needs to be taken while the wafer edge is no longer rounded. Two micro-grating wafers with side etched crystal planes were then joined under an external side force to minimize the joint gap. In this case, the crevice of around $5 \mu \mathrm{m}$ at the interface was observed. With an increasing external side force, the crevice was reduced.

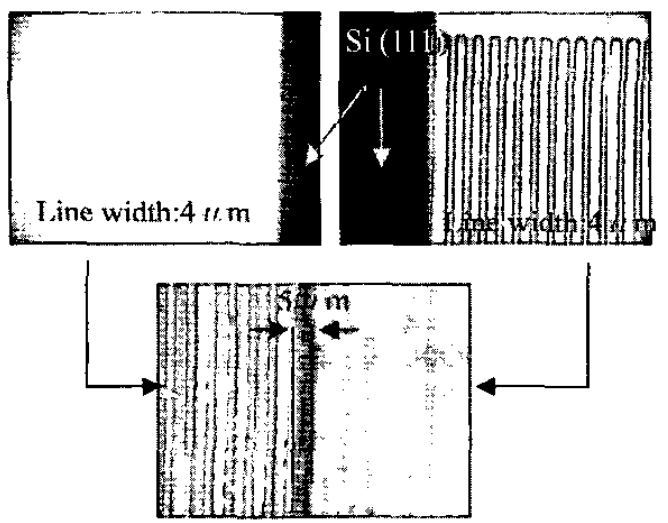

Fig. 6 Lateral joined plates with micrograting structures

The above-mentioned joining silicon plate was then taken as a mold to conduct a hot-embossing process. The use of a silicon substrate as an embossing master possesses many advantages such as excellent hardness, high tensile strength, linear thermal expansion coefficient, good thermal conductivity, versatile geometries readily available, and very flat and even surfaces for effective mold release [1]. A soft pad is crucial for uniform force distribution to cushion pressurized samples against pressing heating plate. The experiment was carried out at embossed temperature $90^{\circ} \mathrm{C}$ and pressure 30 $\mathrm{kgf} / \mathrm{cm}^{2}$. The entire process required 2 minutes in one cycle currently.

In Fig. 7, after the hot embossing onto the PVC plastic sheet, the resultant contracting rate of unit length is 0.0053 . The replication of $99 \%$ from its original master was achieved. The microstructures are faithfully reproduced in the PVC plastic sheet.

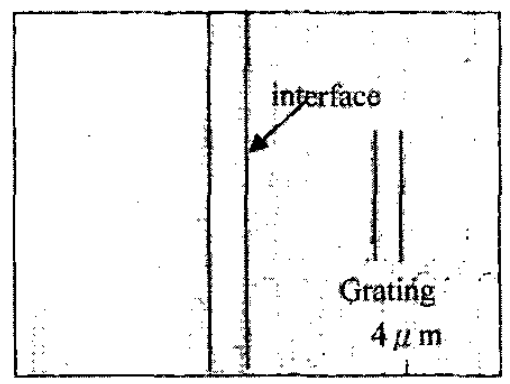

Fig 7 PVC replication by Hot Embossing

In Fig. 8 , the surface profile over the hot-embossed PVC plastic film shows the measured height of $9750 \AA$. After the replication, the transferred surface of its joint plate achieved a sub-micro discrimination. It is also noted that a bump peak of approximate $7 \mu \mathrm{m}$ was produced because in a heating and pressing process the soften PVC surface material flowed into the crevice of the molding plate.

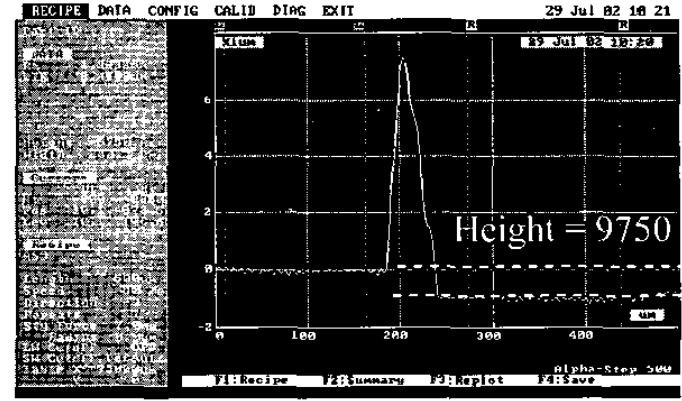

Fig 8 Measured height at the interface of replication

To verify the optical effect of the micro grating structures for the joined plate, the experiment of the diffraction intensities for the zero-order, first-order 
and second-order was carried out by emitting the $\mathrm{He}-\mathrm{Ne}$ laser beam $(633 \mathrm{~nm})$ upon the micro grating structures included with and without the interface. In addition, the simulation grating efficiency using the software, G-Solver, was also conducted to compare with the experimental results. Fig. 9 shows good agreement between the simulation and experiment. Insignificant effect on diffractive optical intensity was observed.

As verified with previous results, Fig. 10 extensively demonstrates its wafer-joining scalability for three processed wafers. The size in area achieved $6.5 \mathrm{~cm} \times 19.5 \mathrm{~cm}$, resembling a one-side length of an 8 -inch silicon wafer. Likewise, with such a lateral joining technique, the plate may potentially expand its one-side into a length of even 12-inch which has first been micromachined in such a sizable scale.

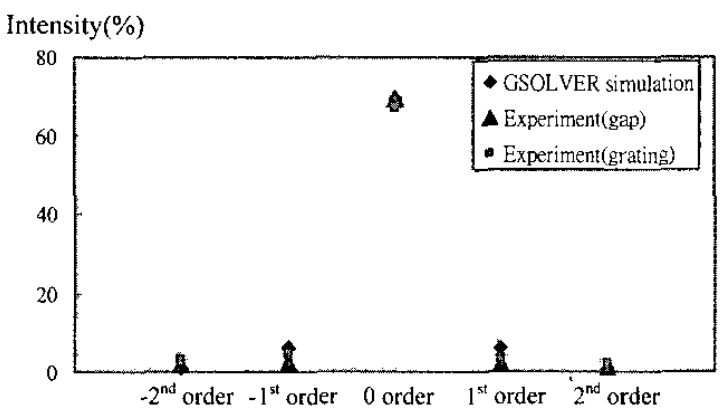

Fig. 9 Comparison of diffraction optical intensities between simulation and experiment.

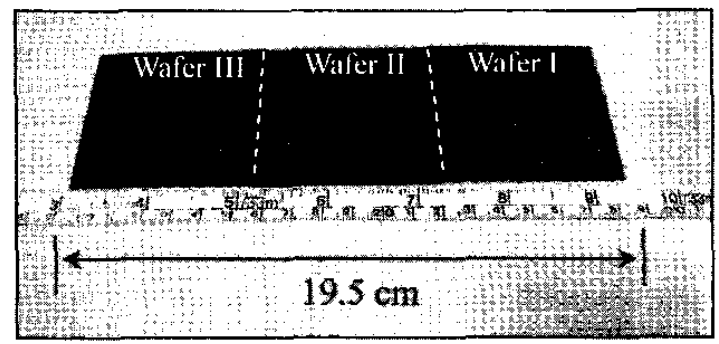

Fig. 10 A large-area silicon plate with micro scale features.

\section{CONCLUSION}

The novel recombining technique using silicon wafer lateral joining has been successfully developed. This silicon-wafer lateral joining technique ensured the gap of several microns, and sub-micron height difference of neighboring joined wafers. The hot embossing process of the joint plate was employed in faithfully replicating the micro grating structures onto the PVC plastic film. Both experimental and simulation results reached good agreement in diffractive intensity efficiency. Insignificant optical intensity effect was observed in the presence of the gap. Furthermore, the joining technique may expand its size with three processed wafers to demonstrate its potential scalability. As a result, the novel technique provides a potentially low-cost approach to fill the technology gap between the conventional precision machining and photolithography-based micromachining for a beyond-wafer-size silicon plate with small features.

\section{REFERENCF}

[1] H. Becker and U. Heim, "Silicon as tool material for polymer hot embossing", MEMS '99, Orlando 1999, pp. 228-232.

[2] M. Heckele, W. Bacher, K.D. Muller, Microsystem Technologies, vol. 4, 1998, pp. 177-182.

[3] Y. Xia, G.M. Whitesides, " Soft lithography" Annu. Rev. Mater. Sc.b, 1998, pp. 153-184.

\section{ACKNOWLEGEMENT}

This work was supported by the National Science Council, Taiwan, R.O.C. under the Grant NSC 90-2218-E-002-026. We would like to express our appreciation to the NSC Northern Regional MEMS Research Center (Taiwan) for fabrication assistance. 